\title{
Piezoelectric nanoelectromechanical resonators based on aluminum nitride thin films
}

\author{
R. B. Karabalin, ${ }^{1}$ M. H. Matheny, ${ }^{1}$ X. L. Feng, ${ }^{1}$ E. Defaÿ, ${ }^{2}$ G. Le Rhun, ${ }^{2}$ C. Marcoux, ${ }^{2}$ \\ S. Hentz, ${ }^{2}$ P. Andreucci, ${ }^{2}$ and M. L. Roukes ${ }^{1, a}$ \\ ${ }^{1}$ Kavli Nanoscience Institute, California Institute of Technology, Pasadena, California 91125, USA \\ ${ }^{2}$ CEA LETI MINATEC, F-38054 Grenoble Cedex, France
}

(Received 22 June 2009; accepted 23 July 2009; published online 9 September 2009)

\begin{abstract}
We demonstrate piezoelectrically actuated, electrically tunable nanomechanical resonators based on multilayers containing a 100-nm-thin aluminum nitride (AIN) layer. Efficient piezoelectric actuation of very high frequency fundamental flexural modes up to $\sim 80 \mathrm{MHz}$ is demonstrated at room temperature. Thermomechanical fluctuations of AlN cantilevers measured by optical interferometry enable calibration of the transduction responsivity and displacement sensitivities of the resonators. Measurements and analyses show that the $100 \mathrm{~nm}$ AlN layer employed has an excellent piezoelectric coefficient, $d_{31}=2.4 \mathrm{pm} / \mathrm{V}$. Doubly clamped AlN beams exhibit significant frequency tuning behavior with applied dc voltage. (C) 2009 American Institute of Physics.
\end{abstract}

[doi:10.1063/1.3216586]

Piezoelectricity, a direct electrical-mechanical conversion occurring in certain types of crystals, has found numerous applications ever since its discovery by the Curie brothers in $1880 .{ }^{1}$ Some of the most ubiquitous among these are electrical oscillators, clocks, and microbalances based on piezoelectric crystals such as quartz. ${ }^{2,3}$ Recent development of micromachining techniques has greatly facilitated the realization of various miniaturized piezoelectric devices, including zinc-oxide $(\mathrm{ZnO})$ beam-structured, ${ }^{4}$ disk, ${ }^{5}$ and film bulk acoustic resonators (FBARs), ${ }^{6}$ the noteworthy Agilent's commercialized AlN FBARs, ${ }^{7}$ AlN contour-mode resonators and filters, ${ }^{8}$ and monolithically integrated FBAR-complementary metal oxide semiconductor (CMOS) filters. ${ }^{9}$ All these piezoelectric microelectromechanical systems (MEMS) have relied on active layers of approximately micron scale thickness that provide strong electromechanical coupling.

The emerging field of nanoelectromechanical systems (NEMS) is attracting considerable interest. These miniaturized nanoscale devices, particularly cantilever and beam flexural-mode resonators, have enabled the demonstrations of single-molecule mass sensors ${ }^{10}$ and single-cell-level force sensors. ${ }^{11}$ For NEMS resonators in such applications, piezoelectric actuation appears to be particularly advantageous compared to the more conventionally employed magnetomotive, ${ }^{10}$ electrostatic, ${ }^{12}$ and electrothermal ${ }^{13}$ excitations. Among its attributes are intrinsic integrability, high efficiency and electrical tunability, low power consumption, and low thermal budgets for materials processing permitting post-CMOS integration. ${ }^{5-9}$ Previously we have prototyped piezoelectric NEMS using epitaxial gallium arsenide (GaAs) heterostructures. ${ }^{14}$ To date, however, nanoscale resonators have not yet been realized with materials having higher piezoelectric coupling efficiency. Here we employ AlN to accomplish this, since it has superior material properties such as high acoustic velocity, $\sqrt{E_{Y} / \rho}$, and low dielectric loss. ${ }^{15}$ Among the principal challenges in its use for NEMS are obtaining nanometer-thick AlN films that retain excellent piezoelectric properties and devising efficient actuation and

${ }^{a)}$ Electronic mail: roukes@caltech.edu. readout schemes. In this letter we demonstrate piezoelectric NEMS resonators actuated by 100 -nm-thick AIN layers, and characterize their driven resonance response, noise spectra, and frequency tuning.

The $100 \mathrm{~nm}$ AlN piezolayer is sandwiched between two $100 \mathrm{~nm}$ molybdenum (Mo) electrode layers. In our $200 \mathrm{~mm}$ wafer-scale process, Mo films are prepared by dc magnetron sputtering, and AlN film is sputtered by a dc pulsed magnetron reactive process. Prior to sputtering the bottom Mo film, a $20 \mathrm{~nm}$ AlN seed layer on a (100) silicon ( $\mathrm{Si}$ ) substrate is deposited. A $99.999 \%$ pure Aluminum (Al) disk is used as the target in $\sim 3$ mTorr of dry nitrogen $\left(\mathrm{N}_{2}\right)$ during sputtering, carried out with $1500 \mathrm{~W}$ of applied power. This process has recently been optimized ${ }^{16}$ to produce highly $c$-axis textured AlN films. Thin film tests indicate that piezoelectric coefficients close to those of bulk AlN can be preserved down to $\sim 250-500 \mathrm{~nm}$ films. ${ }^{17}$

Figure 1 displays a four-mask surface nanomachining process we have developed to fabricate suspended cantilevers and beams using the $\mathrm{Mo} / \mathrm{AlN} / \mathrm{Mo} / \mathrm{seed}-\mathrm{AlN}(100 \mathrm{~nm} /$ $100 \mathrm{~nm} / 100 \mathrm{~nm} / 20 \mathrm{~nm}$ ) stack. A key requirement for piezoelectric actuation is to make electrical contacts to both top and bottom Mo electrodes, as illustrated in Fig. 2(a). In the first step shown in Fig. 1(a), a region is patterned by electron-beam lithography (EBL) using spin-on-glass resist resulting in a $170 \mathrm{~nm}$ silicon dioxide $\left(\mathrm{SiO}_{2}\right)$ "mesa" layer, which protects the structural stack during subsequent processes. Outside of the mesas, argon/chlorine $\left(\mathrm{Ar} / \mathrm{Cl}_{2}\right)$ based inductive coupled plasma (ICP) etch anisotropically removes the top two layers and is terminated at the bottom Mo [Fig. 1(b)]. Gold $\mathrm{(Au}$ ) bonding pads are then patterned and deposited on bottom Mo layer adjacent to mesa regions [Fig. 1(c)]. After parts of the bottom Mo are protected by an insulating $\left(\mathrm{SiO}_{2}\right)$ bridge, the remaining is removed by a wet etch, yielding the device profile shown in Fig. 1(c), with bottom contact electrode displayed on the left and top electrode on the right. This separate patterning of both top and bottom bonding pads on the same Mo layer substantially reduces parasitics.

After stripping the $\mathrm{SiO}_{2}$, the mesa's top Mo layer is exposed and another $\mathrm{SiO}_{2}$ bridge with conducting path on top 

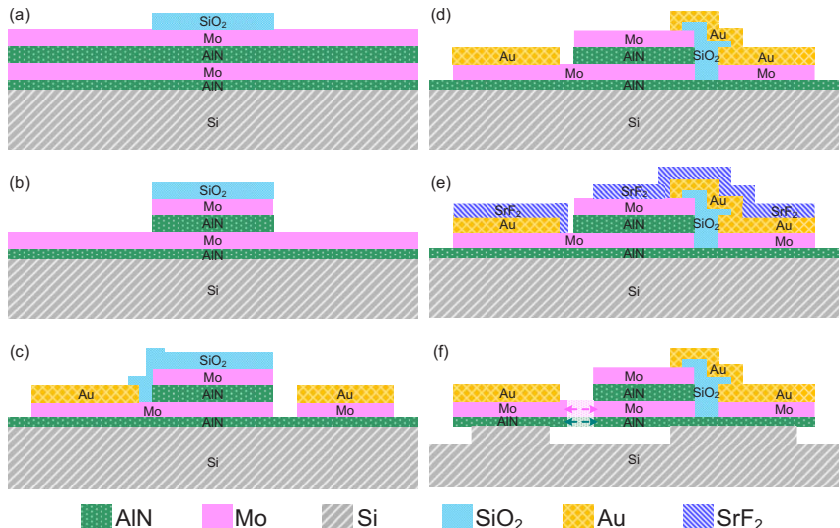

FIG. 1. (Color online) Surface nanomachining process flow for piezoelectric AlN NEMS. (a) Definition of $\mathrm{SiO}_{2}$ mesa, followed by a dry etch (b) to remove top Mo and AlN. (c) Au electrodes are patterned and deposited adjacent to the mesa region, along with an $\mathrm{SiO}_{2}$ bridge protecting part of bottom Mo, while the remaining bottom Mo was removed. (d) $\mathrm{SiO}_{2}$ is stripped and contact is made to the top Mo electrode (including deposition of a $\mathrm{SiO}_{2}$ bridge layer). (e) NEMS devices are defined, using $\mathrm{SrF}_{2}$ as a dry etch mask for both NEMS and all metallic contacts. (f) Anisotropic etching of all the structural layers down to $\mathrm{Si}$ by using $\mathrm{Ar} / \mathrm{Cl}_{2}$ ICP-RIE is followed by an isotropic $\mathrm{Ar} / \mathrm{NF}_{3}$ etch for device release, and subsequent removal of $\mathrm{SrF}_{2}$ mask. The dashed arrows and lightly hatched areas indicate that the bottom AlN/Mo layers are connected out of the present section plane.

is patterned between the mesa and top electrode [Fig. 1(d)]. As shown in Fig. 1(e), the nanodevices are then patterned with high resolution EBL followed by deposition of an insulating strontium fluoride $\left(\mathrm{SrF}_{2}\right)$ mask. An anisotropic ICP etch is used to transfer the pattern through all four structural layers, down to the Si substrate. Finally, devices are suspended by isotropically etching the Si sacrificial layer in an
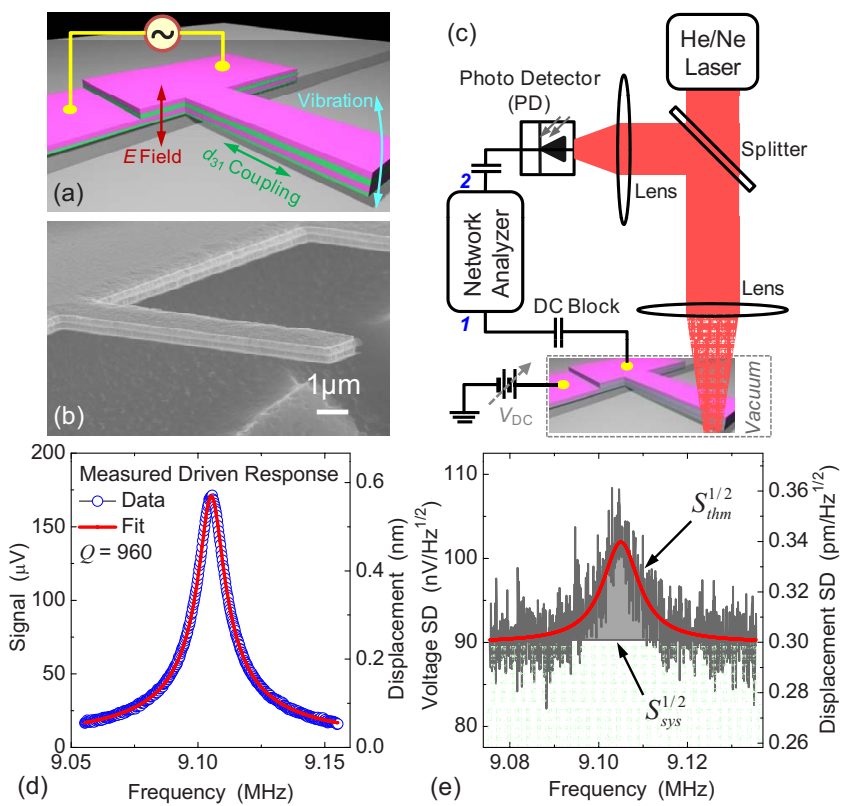

FIG. 2. (Color online) Characterization of high frequency AlN cantilever NEMS. (a) Illustration of piezoelectric actuation. (b) An SEM image displaying the four-layer composite structure of a cantilever NEMS. (c) Simplified schematic of the optical readout scheme (note for the undriven noise measurements, port 1 is disconnected and network analyzer is replaced by a spectrum analyzer; also $V_{\mathrm{dc}}$ is applied only during the tuning measurements shown in Fig. 3). (d) A typical resonant response measured from a driven cantilever [device shown in (b)], with $f_{0}=9.11 \mathrm{MHz}$ and $Q=960$, extracted from the fit to the model of damped driven oscillator. (e) Measured thermomechanical noise spectral density from the $9.11 \mathrm{MHz}$ device, demonstrating the sensitivities in both electrical and mechanical domains. argon/nitrogen trifluoride $\left(\mathrm{Ar} / \mathrm{NF}_{3}\right)$ plasma. The scanning electron micrograph (SEM) of a typical device [Fig. 2(b)] shows the composing layers in the structure.

When a voltage signal is applied between the device's top and bottom electrodes [Fig. 2(a)], the piezoelectricity tensor causes longitudinal strain to develop in the active layer according to $s_{x x}=d_{31} E_{z}$, where $s_{x x}$ is the piezoelectric longitudinal strain, $E_{z}$ is a vertical component of electric field, and $d_{31}$ is the piezoelectric coefficient. The seed layer offsets the neutral plane from the active layer's central plane; as a result, when the piezoelectric layer is strained, it causes a bending moment in the structure. Upon application of highfrequency actuation, devices are driven into resonance and their response is characterized via two-port network analysis. In our specific transduction scheme, the out-of-plane fundamental flexural-mode resonances are usually most prominent. In order to avoid crosstalk between actuation and detection signals we use an optical interferometric technique ${ }^{18}$ to readout the mechanical displacement [Fig. 2(c)]. All measurements are performed at room temperature at a base pressure of $\sim 5$ mTorr. Figure 2(d) shows the measured resonance of a typical AlN cantilever [Fig. 2(b)] with dimensions of $L \times w \times t=6 \mu \mathrm{m} \times 900 \mathrm{~nm} \times 320 \mathrm{~nm}$. A Lorentz fit to the measured response yields a quality factor $Q=960$ for the fundamental resonance at $9.11 \mathrm{MHz}$. The observed resonance frequency closely matches our analytical prediction $(9.05 \mathrm{MHz})$ for the four-layered cantilever, given by ${ }^{19}$

$$
f_{0, t h}=\frac{(1.875)^{2}}{2 \pi L^{2}}\left[\sum_{i=1}^{4} E_{Y i} w_{i} \int_{\substack{\sum_{n=1} t_{n} \\ \sum_{n=1}^{i} t_{n}}}^{i}\left(z-z_{c}\right)^{2} d z / \sum_{i=1}^{4} \rho_{i} w_{i} t_{i}\right]^{1 / 2},
$$

where

$$
z_{c}=\sum_{i=1}^{4} E_{Y i} \int_{\substack{\sum_{n=1}^{i-1} t_{n} \\ \sum_{n=1}^{i} t_{n}}}^{i} z d z / \sum_{i=1}^{4} E_{Y i} t_{i} .
$$

Here $z_{c}$ is the position of the structure's elastic neutral plane ( $z=0$ is at the bottom of the multilayer). The indices correspond to four consecutive layers of the material: (1) seed AlN, thickness $t_{1}=20 \mathrm{~nm}$, Young's modulus $E_{Y 1}=345 \mathrm{GPa}$; (2) bottom Mo, $t_{2}=100 \mathrm{~nm}, E_{Y 2}=329 \mathrm{GPa}$; (3) active AlN, $t_{3}=100 \mathrm{~nm}$; and (4) top Mo with $t_{4}=100 \mathrm{~nm}$.

The displacement sensitivity of our readout scheme is sufficient to detect the device's thermomechanical noise. We obtain the noise spectra of the undriven devices using a spectrum analyzer. Figure 2(e) demonstrates the thermomechanical noise spectrum of the $9.11 \mathrm{MHz}$ cantilever. In the limit of $Q \gg 1$, the thermomechanical displacement noise spectral density on resonance is $S_{z, \text { thm }}^{1 / 2}=\left[k_{B} T Q /\left(2 \pi^{3} f_{0}^{3} M_{\text {eff }}\right)\right]^{1 / 2}$, where $k_{B}, T$, and $M_{\text {eff }}$ are Boltzmann's constant, temperature, and the device's effective mass, respectively. This yields the room-temperature displacement noise spectral density on resonance, $S_{z, \text { thm }}^{1 / 2} \approx 0.16 \mathrm{pm} / \mathrm{Hz}^{1 / 2}$. The spectrum in Fig. $2(\mathrm{e})$ has a background noise floor $S_{v, \text { sys }}^{1 / 2} \approx 90 \mathrm{nV} / \mathrm{Hz}^{1 / 2}$ near the resonance. The thermomechanical displacement noise density is transduced into a voltage noise density $S_{v, \text { thm }}^{1 / 2}$ $=\left(S_{v \text {,total }}-S_{v, \text { sys }}\right)^{1 / 2} \approx 48 \mathrm{nV} / \mathrm{Hz}^{1 / 2}$. From this analysis we determine a transduction responsivity $\mathfrak{R} \equiv S_{v, \text { thm }}^{1 / 2} / S_{z, \text { thm }}^{1 / 2}$ $\approx 300 \mathrm{nV} / \mathrm{pm}$, and a mechanical displacement sensitivity $S_{z, \mathrm{sys}}^{1 / 2}=S_{v, \mathrm{sys}}^{1 / 2} / \mathfrak{R} \approx 0.3 \mathrm{pm} / \mathrm{Hz}^{1 / 2}$ for the measurement system. 

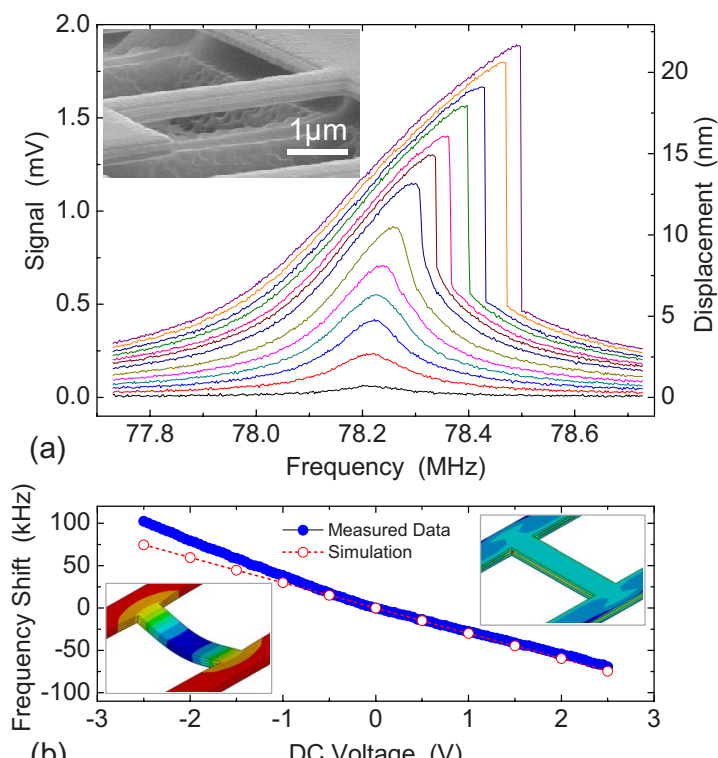

(b)

DC Voltage (V)

FIG. 3. (Color online) VHF AlN beam resonators demonstrating nonlinearity and frequency tuning behavior. (a) The measured $78.2 \mathrm{MHz}$ resonance clearly manifests the Duffing nonlinearity as the excitation is increased. Inset is an SEM image of the device employed. (b) Measured and computed piezoelectric frequency tuning as a function of dc polarization voltage across the sandwiched AlN layer. Insets illustrate the static longitudinal strain distribution (upper right) induced by a $2.5 \mathrm{~V}$ dc polarization, and the mode shape (lower left) corresponding to the measured resonance.

With this calibration of the transduction responsivity, we map out device's motions in the mechanical domain. For the response plotted in Fig. 2(d), the displacement on resonance is $0.54 \mathrm{~nm}$ at $2 \mathrm{mV}$ drive. Following a detailed numerical analysis of piezoelectrically deflected multilayer cantilevers, ${ }^{20}$ we determine the piezoelectric coefficient of the $100 \mathrm{~nm}$ AlN layer to be $d_{31}=2.4 \mathrm{pm} / \mathrm{V}$. To complement these measurements, we have also fabricated centimeterscale samples from the same wafer, and independently measured the piezoelectric constant of the AlN layer by means of a setup detailed elsewhere. ${ }^{21}$ Such measurements yield $d_{31}=2.6 \mathrm{pm} / \mathrm{V}$, a value consistent with that determined by the NEMS resonance measurements. These values $(\sim 2.5 \mathrm{pm} / \mathrm{V})$ are only slightly lower than those reported for single crystals. ${ }^{17}$ This demonstrates the high performance that can be attained with $100 \mathrm{~nm}$ polycrystalline AIN films.

We further demonstrate operation of tunable, doubly clamped beam AIN resonators. In beams such as displayed in the SEM inset of Fig. 3(a), the piezoelectric effect not only generates actuation but also produces resonance frequency tuning by application of a dc voltage. This particular device has dimensions $L \times w \times t=4 \mu \mathrm{m} \times 900 \mathrm{~nm} \times 320 \mathrm{~nm}$, resonance frequency $f_{0}=78.2 \mathrm{MHz}$, and quality factor $Q=670$. The moderate $Q$ reduction (compared to the $9.11 \mathrm{MHz}$ cantilever) for this smaller device with larger surface to volume ratio is consistent with earlier observations ${ }^{22}$ of a $Q$ dependence on the size of MEMS/NEMS resonators, likely due to relatively larger clamping and surface losses. Resonance curves measured with increasing drive amplitudes [Fig. 3(a)] show that with modest excitation voltages, motion beyond the onset of nonlinearity ${ }^{23}$ is obtained. These measurements agree with analytic predictions of the Duffing nonlinearity, which arises from induced tension in doubly clamped beam resonators. ${ }^{24}$
As a dc voltage is applied between the top and bottom electrodes, an electrical field is created across the AlN layer, and the piezoelectrically induced strain is converted into a dc longitudinal stress, subject to the clamped-clamped boundary conditions. This modulation of the tensile stress leads to a shift in resonance frequency. This behavior is verified by monitoring the resonance frequency of a beam while sweeping the dc voltage. Figure 3(b) shows the measured frequency tuning as a function of the applied dc voltage. As expected, the resonance frequency varies approximately linearly with the voltage. The measured frequency tunability of $34 \mathrm{kHz} / \mathrm{V}$ is consistent with results from finite element simulations, plotted in Fig. 3(b), where multiple layers and finite ledges $(1 \mu \mathrm{m}$ in width, resulting from the undercut associated with device release) are taken into account.

We demonstrate piezoelectric very high frequency tunable NEMS resonators, which offer significant potential for a broad spectrum of applications.

We are grateful to G. Villanueva, J.L. Arlett, and J.E. Sader for helpful discussions and Y. Wu for illustration. We acknowledge financial support for this work from DARPA/ MTO and SPAWAR under the Grant No. N66001-07-1-2039.

${ }^{1}$ P. Curie and J. Curie, Bull. Soc. Fr. Mineral. 3, 90 (1880).

${ }^{2}$ W. G. Cady, Piezoelectricity: An Introduction to the Theory and Applications of Electro-Mechanical Phenomena in Crystals (Dover, New York, 1964).

${ }^{3}$ Applications of Piezoelectric Quartz Crystal Microbalances, edited by C. Lu and A. W. Czanderna (Elsevier, New York, 1984).

${ }^{4}$ D. L. DeVoe, Sens. Actuators, A 88, 263 (2001).

${ }^{5}$ L. Yan, W. Pang, E. S. Kim, and W. C. Tang, Appl. Phys. Lett. 87, 154103 (2005).

${ }^{6}$ H. Zhang and E. S. Kim, J. Microelectromech. Syst. 14, 699 (2005).

${ }^{7}$ R. C. Ruby, P. Bradley, Y. Oshmyanksy, A. Chien, and J. D. Larson III, Proc.-IEEE Ultrason. Symp. 1, 813 (2001).

${ }^{8}$ G. Piazza, P. J. Stephanou, and A. P. Pisano, J. Microelectromech. Syst. 16, 319 (2007).

${ }^{9}$ M.-A. Dubois, J.-F. Carpentier, P. Vincent, C. Billard, G. Parat, C. Muller, P. Ancey, and P. Conti, IEEE J. Solid-State Circuits 41, 7 (2006).

${ }^{10}$ Y. T. Yang, C. Callegari, X. L. Feng, K. L. Ekinci, and M. L. Roukes, Nano Lett. 6, 583 (2006).

${ }^{11}$ J. L. Arlett, J. R. Maloney, B. Gudlewski, M. Muluneh, and M. L. Roukes, Nano Lett. 6, 1000 (2006).

${ }^{12}$ R. R. He, X. L. Feng, M. L. Roukes, and P. D. Yang, Nano Lett. 8, 1756 (2008).

${ }^{13}$ I. Bargatin, I. Kozinsky, and M. L. Roukes, Appl. Phys. Lett. 90, 093116 (2007)

${ }^{14}$ S. C. Masmanidis, R. B. Karabalin, I. De Vlaminck, G. Borghs, M. R. Freeman, and M. L. Roukes, Science 317, 780 (2007).

${ }^{15}$ K. Tsubouchi, K. Sugai, and N. Mikoshiba, Proc.-IEEE Ultrason. Symp. 1981, 375 .

${ }^{16}$ F. Martin, P. Muralt, and M. A. Dubois, J. Vac. Sci. Technol. A 24, 946 (2006).

${ }^{17}$ F. Martin, P. Muralt, M. A. Dubois, and A. Pezous, J. Vac. Sci. Technol. A 22, 361 (2004)

${ }^{18}$ D. W. Carr and H. G. Craighead, J. Vac. Sci. Technol. B 15, 2760 (1997).

${ }^{19}$ R. J. Roark and W. C. Young, Formulas for Stress and Strain (McGrawHill, New York, 1975).

${ }^{20}$ J. G. Smits and W. S. Choi, IEEE Trans. Ultrason. Ferroelectr. Freq. Control 38, 256 (1991).

${ }^{21}$ E. Defay, C. Zinck, C. Malhaire, N. Baboux, and D. Barbier, Rev. Sci. Instrum. 77, 103903 (2006).

${ }^{22}$ P. Mohanty, D. A. Harrington, K. L. Ekinci, Y. T. Yang, M. J. Murphy, and M. L. Roukes, Phys. Rev. B 66, 085416 (2002).

${ }^{23}$ A. H. Nayfeh and D. T. Mook, Nonlinear Oscillations (Wiley, New York, 1979).

${ }^{24}$ H. W. C. Postma, I. Kozinsky, A. Husain, and M. L. Roukes, Appl. Phys. Lett. 86, 223105 (2005). 